\title{
Drug pathways, transitions and decisions: The experiences of young people in an inner-city Dublin community
}

\author{
BY PAULA MAYOCK
}

This paper reports on selected findings from an ethnographic study of drug use by young people in a Dublin inner-city community considered to be "high risk" for problem drug use. In-depth individual interviews and focusgroup discussions were used to generate extensive data on the social and drug-related experiences of young people ages 15-19 years. Fifty-seven young people were interviewed individually, and a subsample of 24 took part in focus-group discussions. The paper explores young people's drug "journeys" and documents emerging drug pathways. It examines the processes relevant to young people's drug transitions, drawing attention to the role of risk perceptions and risk boundaries in decision-making about drugs. The findings highlight the role of social/contextual influences in the onward transitions to new drugs and suggest that drug choices are strongly mediated by young people's experience of, and interaction with, their social environment.

KEY WORDS: Drugs, young people, drug decisions, risk perceptions, Ireland.

AUTHOR'S NOTE: The data reported in this paper were collected when the author worked at the Children's Research Centre, Trinity College, Dublin.

(C) 2002 by Federal Legal Publications, Inc. 
In Ireland, as elsewhere, drug use by young people has moved from peripheral subcultures to a situation where drugs appear to be increasingly accessible and more widely used. ${ }^{1}$ Results from the most recent national study of tobacco, alcohol and drug use by adolescents indicate that $32 \%$ of 16-year-olds have tried cannabis and 9\% have used another drug besides cannabis in their lifetime (Hibell et al., 2000). ${ }^{2}$ Despite increased recognition of the widespread nature of drug use among young people, the bulk of research in an Irish context has focused on establishing reliable estimates of the extent of opiate use (Comiskey, 1998) and on generating accurate profiles of problem drug users who seek treatment (O'Higgins. 1996; O'Higgins \& Duff, 1997; O'Brien et al., 2000). ${ }^{3}$ One of the most consistent findings to emerge from this work concerns the link between concentrations of drug problems and high levels of social and economic deprivation (Dean et al., 1983; O'Kelly et al., 1988; McKeown et al., 1993; Comiskey. 1998). However, little is known about the social and drug-related experiences of young people living in localities identified as having a history of concentrated drug problems. In short, we have relatively little knowledge or understanding of how young people negotiate "risky" environments. This represents a serious gap in knowledge, given the influential role of the environmental conditions in the development and maintenance of commitments to drug use (ACMD, 1998).

A considerable range of research approaches and methods have been employed in an effort to establish key factors underlying substance use among young people. Risk-factor research has played a major role in identifying a wide range of environmental, behavioral, psychological and social variables associated with drug use (see Hawkins et al., 1992, for review). One of the difficulties, however, with the risk-factor approach is its tendency to regard individuals as essentially passive recipients of "influence" without considering the complex nature of individual and group responses or the social processes involved in the negotiation of "risk." The epidemiological emphasis on actualized risk neglects the 
critical issue of how risk is perceived and negotiated by individual users and non-users of a range of psychoactive substances. Furthermore, it tends to isolate drug use from important aspects of young people's lives, thereby neglecting the sociocultural context in which drug use occurs. More recently, the processes influencing young people's substance-related behavior have received greater attention, and with this, attention has focused on the issue of drug-related decision-making. Research has, for example, highlighted the differential ways in which drugs are used and has documented ways in which young people alter and modify their drug-related behavior over time (Measham et al., 1998; Bell et al., 1998). Other research has documented the rejection of certain drugs by young people despite opportunities for use (Glassner \& Loughlin, 1987; Fountain et al., 1999). Boys et al.'s (1999) qualitative study of young people's drug use uncovered individual-level and social/contextual-level influences on drug use and identified the perceived function of using particular substances as playing an influential role in drug-related decisions. Other studies similarly suggest that decisions about the use of drugs are related to individual appraisals of benefit versus risk, rather than being a passive response to opportunities and/or incentives for use (Parker et al., 1998; Boys et al., 1999; Mayock, 2000a; Wibberley \& Price, 2000).

The social context of drug use, including individual and group subjective interpretations of drug use and the settings in which drug use occurs, is viewed as a key process influencing how drug use practices are socially organized (Rhodes, 2000). Recent research concerned with substancerelated decision-making by young people has focused heavily on users' motives for use and has drawn attention to the importance of recognizing the function(s) or specific purposes that individual substances serve within a variety of settings (Boys et al., 1999; Boys et al., 2001). Other research has illustrated how young people adhere to "rules" concerning the use of drugs, suggesting that young drug users have established 
boundaries beyond which they will not go (Glassner \& Loughlin, 1987). However, despite considerable evidence of movement "into" and "out of drug use of various kinds and at variable levels of use frequency throughout the teenage years (Measham et al., 1998), the processes influencing young people's drug transitions remain largely unexplored. In particular, little attention has focused on how risk perceptions impact on transitions to new drugs and/or changed patterns of use. This paper reports on selected findings from an ethnographic study of drug use by young people and examines young people's drug decisions by drawing attention to key transitional points in their drug "journeys." Young people's drug taking is examined within a framework that highlights the complexity of their differential involvement with, and rejection of, psychoactive substances. A close examination of how young people describe their movement to new drugs and across recognized risk boundaries draws attention to the situatedness of drug decisions and highlights the role of the individual actor, within a range of influences, in the decision to use or not to use certain, or any, illicit drugs. The paper closes by discussing some of the key insights and lessons arising from (he research and relates these to recent drug-policy developments in an Irish context.

\section{Methodology}

One of the primary aims in undertaking this research was to provide detailed knowledge and understanding of the range and types of drug taking evidenced by a sample of young people in their mid- to late-teenage years. This was achieved through close contact, participation and collaboration with young people in their natural settings - that is, in the area where they live. The research approach, involving two separate periods of active engagement within the research site, embodied strong ethnographic qualities. The initial phase of fieldwork, characterized by regular (daily) contact with young peoplewithin the research site, was conducted in 1998. The 
second phase of the research, only recently completed, involved "tracking" and re-interviewing a large number of the study's participants following a three-year time lapse. ${ }^{4}$ The findings documented in this paper are drawn from data collected during the first phase of the study.

The research locality—a Dublin inner-city community—endured a local heroin epidemic during the 1980s and continues to host a large heroin-using population. The neighborhood is considered to have one of the most serious drug problems in the State and was designated for inclusion in the Irish Government's Local Drugs Task Force Initiative as pan of a wider strategy aimed at tackling drug problems at local level (First Report of the Ministerial Task Force on Measures to Reduce the Demand for Drugs, 1996). The study's participants are a group considered to be "high risk" for drug involvement by virtue of living in an area characterized by social deprivation coupled with high levels of drug exposure (ACMD, 1998; Crum et al., 1996; Lloyd, 1998). The recruitment of respondents, largely a social endeavor, took place within the community. Young people were contacted within a range of settings, including youth clubs, local drug services, satellite clinics, drop-in centers, and numerous outdoor locations where young people congregate. A variety of techniques, including "snowballing" (van Meter, 1990) and "targeted" sampling (Walters \& Biernacki, 1989), were used in an effort to access young people thought to have differing opportunities for, and experiences of, drug use. As time progressed, specific strategies were developed in order to access more "hidden," resistant and "difficult to reach" young people who had little contact with local youth clubs and other community-based recreational facilities (Mayock, 2000b). The research strategy, characterized by active participation within the young people's social milieu, provided access to a repertoire of experiences and facilitated an understanding not only of their drug use, but also of the broader context in which their experiences are situated. 
Individual in-depth interviews were used to elicit detailed information on a range of topics, including demographics, education and employment, druguse history, current drug use, peer relationships and peer drug use, stressful life events, family relationships, drug attitudes, motives for use/non-use, knowledge of the local drug scene, and perceptions of community life. All interviews were tape recorded and varied in duration from 90 to 150 minutes. Focus groups were used to explore broader issues pertaining to young people's social experiences, their drug knowledge and drug attitudes, and their perceptions of the area where they lived. These discussions acted as an exploratory tool by uncovering opinions, attitudes and so forth throughout the fieldwork process. ${ }^{5}$ Finally, demographic details were recorded for each individual informant, using a short questionnaire. Baseline data on respondents' drug-taking history was recorded by asking each young person to indicate the drugs they had "ever used," those used during "the past year" and "the past week," and those they "may use in the future."

The selection process aimed to include a range of drug-taking experiences and, unlike previous studies undertaken in areas hosting a large number of "known" drug users (Pearson et al., 1985; Parker et al., 1988), did not confine itself to the experiences of young heroin users. The research focused on non-use, drug use not defined as problematic, and drug use defined as problematic by the young people under study. Three categories of research respondents-abstainers, drug-takers, and problem drugtakers-were recruited for participation. Young people 15-19 years old were the target group for the research, as it was felt that this age group is particularly susceptible to drug use at some level (Kandel \& Logan, 1984; Measham et al., 1994). ${ }^{6}$ The following definitions were applied to each of the three participating groups of respondents at the outset of the study:

Abstainers: Young people who are not using drugs at present. They may have experimented with a soft drug-e.g., 
cannabis - at some stage but must have not done so for a minimum of six months.

Drugtakers: Young people who use drugs for recreational or experimental purposes. Frequency of use varies among this group, as do the type and number of drugs used. In recognition of the widespread availability of stimulants and amphetamine-based drugs, young people who experiment with or use these occasionally are included in this category. These young people do not consider their drug use to be problematic.

Problem drugtakers: Young people who experience difficulties as a result of their drug taking. They may be dependent on opiates (heroin, methadone) or other drugs (stimulants, cannabis) and may or may not be receiving treatment at present. These young people consider their drug use to be problematic.

This "categorization" of young people emerged through a process of selfnomination. In other words, the views and attitudes of the young people, not those of the researcher or other professionals, determined participants' drug status - be it abstainer, drugtaker, or problem drugtaker-within the parameters of the study. This was achieved through questioning and was based on young people's perceptions of the risks, benefits, effects and consequences of their drug use. This approach, with its emphasis on the socially constructed nature of reality, precluded the imposition of "outsider" judgments about the nature and consequences of informants' drug use.

Fifty-seven young people (33 females and 24 males) were interviewed individually, and a subsample of 24 participants (14 females and 10 males) took part in focus-group discussions. The mean age of research participants was 17.3 years at the time of conducting the first phase of fieldwork. Across the sample, the overall picture was one of substantial socio-economic disadvantage. The brunt of this disadvantage 
appeared to fall on young people in the drugtaking and problem drugtaking categories, who were more likely than abstainers to be living in local authority flat complexes, to have left school early and without formal qualifications, and to be unemployed or only casually employed. Drugtakers and problem drugtakers were less likely than abstainers to be living in twoparent family homes and to enjoy the benefit of additional income from parents in either full- or part-time employment.

\section{Study findings}

This section opens by presenting descriptive data on the study locality, with particular attention to typical accounts of first drug-taking experiences. The focus moves then to respondents' reported drug use, highlighting considerable movement between categories and sub-categories across the sample. Young people's drug pathways are examined by identifying the processes relevant to their drug transitions from the point of initial use. The distinctions made between drugs and among drug users and non-users, with particular attention to differential risk perceptions, form the basis of a later analysis of young people's drug decisions.

\section{The social environment}

The research site has a lengthy history as a locality where drugs are bought and/or consumed and has been associated with concentrations of drug problems since the early 1980s (Dean et al., 1983; Cullen, 1991). High levels of street dealing and extensive media coverage of the phenomenon have contributed to the area's notoriety as a "hot spot" for drug acquisition. Furthermore, specific pockets or sub-localities in the neighborhood have been associated with intense drug dealing and distribution activity for some time. 
The presence of a thriving drug scene emerged strongly from the young people's reports. The majority made constant reference to the sale and use of drugs when offering routine descriptions of community life. Respondents' accounts suggest that heroin and cannabis, followed by tranquilizers, amphetamine. Ecstasy and street methadone, were the most accessible drugs. Other substances, including cocaine and LSD, appeared to be less visible, with supplies perceived as sporadic and access routes dependent to a greater extent on individuals' personal connections to dealers. While some young people expressed strong dissatisfaction with their constant exposure to the visible signs of heroin and other serious drug use, others viewed drugs rather resignedly as part and parcel of everyday life. The routine nature of drug encounters emerged strongly from the young people's descriptions of initial drug use, an event that was invariably reported in a matter-of-fact way: "Can't really remember [first drug-taking event], ah yeah, I do, I was in one of the Blocks with me sister's mate, and he asked me did I want to try it, and I said 'Yeah.',

The majority of young people reported a whole range of situations, including the street, the schoolyard, and routine social gatherings with peers, where drugs were easily available. Friends, not adult dealers, were the principal suppliers of first drugs, and there was strong evidence to suggest a fusion of user/dealer boundaries, particularly in relation to the supply and acquisition of cannabis. As one participant casually remarked, "Some of the young fellas out of the flats sell hash, like." A young woman who reported daily cannabis use explained her involvement in cannabis dealing by making a clear distinction between the local heroin and cannabis markets: "I always sell the hash, ya know what I mean. Like, it's a risk you're takin' but everyone knows . . . like, most know like, that's the gear [heroin] Block an' I don't be over there. Like, I don't sell hash all the time, just when I have no money." 
Drug initiation occurred at an early age-13.3 years for drug-takers and 12.4 years for problem drugtakers. Reports of initial use indicate that first drug experiences often transpired by chance. The event generally took place in the company of friends, and cannabis was invariably the first drug tried. The presence of peers was important for several reasons. First, friends supplied cannabis, the most commonly used first drug. More important, their presence meant the experience was shared, creating a sense of security or "safety" around the event. The quotations below help to illustrate the casual and incidental nature of first drug encounters.

Hash was the first drug I tried. ... I got it off a fella in school, a fella I used ta hang around with in school. Yeah, he had it there like, he had it rolled an' all an' he lit up and said, "Do you wanna drag?" so 1 just took a drag off it. (Mark, 17.1 years)

I was with me friends and they said they had enough for a joint. And they said did I want it and I said, "Sure, I'll try it." (Crystal, 15.5 years)

Drugs were very much "around," and young people were well acquainted with the notion of drug use prior to their first drug-taking experience. Moreover, the narratives strongly suggest that the drug scene was regarded as an enduring feature of community life. Drug use and drug trafficking were highly visible, and it was common to see groups of young people congregated at certain sites in the neighborhood clearly marked out for drug use. Cannabis, in particular, was smoked without a high level of concealment, although it was noticeable that young people did maintain a level of discretion around all drug use, particularly when preparing drugs for use. $^{7}$ One could justifiably conclude that the young people interviewed live amid a thriving drugs culture.

\section{Drug pathways}

While uniformity emerged in the descriptions offered of first drug-taking, reported patterns of use subsequent to first and 
early drug experiences presented a far more complex picture. It is helpful, therefore, to summarize some of the distinguishing features of the types and levels of drug involvement evidenced across the sample.

First, enormous variation emerged both within and among the three participating groups of research respondents in terms of the number, type and frequency of drug use. Hence, while abstainers, drugtakers and problem drugtakers broadly represent differing levels of commitment to drug use, considerable variation emerged in the drug-taking practices reported within all three participating "categories" of informants. Second, the drug-taking practices reported by the study's drug users, including those of drugtakers and problem drugtakers, did not remain stable across time. Young people described movement from one drug to another, and their drug preferences altered conspicuously, alongside "new" knowledge and experience of a range of substances. Several, for example, reported discontinuing certain forms of drug use following a period of experimentation. Others reported significant modification to their drug intake. The experiences of abstainers, as will be demonstrated later, also confirm that drug use, rather than being stable or fixed, is in a constant state of flux during the teenage years.

Finally, the two drug-using categories of respondents-drug-takers and problem drugtakers - differed markedly in their level of immersion in the drug scene and in their depth of involvement with a range of substances. They also differed in terms of the perceived difficulties arising from their drug consumption. Whereas drugtakers did not consider their drug use to be problematic, problem drugtakers reported serious negative physical, social and psychological consequences arising from their drug consumption. The two groups also differed in their experience and use of "hard" drugs, heroin and cocaine. While it was unusual for drugtakers to have tried opiates, the vast majority of problem drugtakers reported 
difficulties related to their heroin and/or other drug consumption.

Before examining the variable accounts of drug use, it is helpful to provide an overview of the range of drugs tried and used by the study's selfconfirmed drug users. Table 1 presents data pertaining to lifetime drug use ${ }^{8}$ for drugtakers and problem drugtakers.

TABLE 1 Lifetime drug use: drugtakers and problem drugtakers

Cannabis

\begin{tabular}{cc|}
\multicolumn{2}{c}{$\%$ Lifetime Use } \\
Drugtakers & Problem Drugtakers \\
$(\mathrm{n}=21)$ & $(\mathrm{n}=18)$ \\
100 & 100 \\
48 & 88 \\
43 & 75 \\
62 & 69 \\
10 & 88 \\
10 & 81 \\
19 & 19 \\
41 & 81 \\
29 & 75 \\
5 & 81
\end{tabular}

The most striking feature here is the high level of drug-trying. Cannabis dominated as the drug first tried. It was also the most popular and frequently used drug: $85 \%$ of the drugtakers and $90 \%$ of problem drugtakers intended to use cannabis during the week following the interview. The drugs Ecstasy, amphetamine (speed), LSD (acid), and tranquilizers were used extensively by research respondents. Not surprisingly, a greater proportion of problem drugtakers reported lifetime use of all the illegal substances, and all reported problems associated with heroin and/or other drug use.

While the figures presented above are useful in summarizing the range of drugs ever used, they provide little insight into young people's mode or "style" of engagement with a range 
of psychoactive substances. This information was generated from a detailed analysis of respondents' drug histories. The variable levels of drug involvement uncovered suggest a continuum of commitment to drug use. Furthermore, numerous drug pathways emerged, confirming the variable processes and dynamics influencing young people's drug journeys. The reports of abstainers play an important role in this analysis, providing considerable insight into how young people navigate an environment where there are ample opportunity and incentive for drug use. The following sections examine the drug-taking experiences of abstainers, drugtakers, and problem drugtakers in greater detail.

Abstainers The drug-related experiences of abstainers are rather more diverse than might have been anticipated. Of the 18 abstainers interviewed, six reported a drug history. Among those who reported prior drug use, the general picture was one of experimentation associated primarily with curiosity and peer gatherings involving the use of drugs.

[So, who had the hash?] One of me mates, he was just trying it out, we all were ... we were up there under the bridge at the canal, about seven of us. [And how did it make you feel?] I felt sickish and I had a dry feelin' in my mouth. (Paul, 16.4 years)

All six former drug triers had tried cannabis at some stage, and two had sampled both Ecstasy and amphetamine. For most, use was experimental. However, two young people stated that they had smoked cannabis regularly at some time in the past. One such respondent offered the following account:

[Can you tell me about smoking hash when you were younger?] I was about 13. I used to buy it in the flats. There was three of us that used to buy it together and between us we used ta smoke, well, it got to $15 £$ worth a day. We used to be mad out of it ... [What made you stop?] One night I woke up and I thought I was gonna die, I couldn't breathe ... I could breathe but I thought I couldn't, so I sat up all night and I just never touched it again after that. [So, that was the main reason you stopped smoking?] Well, kind of, yeah. But 1 just got pissed off with it as well, like, 'cos it was get 
ting' to the stage like, $15 £$ worth a day, and then we weren't getting a buzz outa that, ya know what 1 mean. And I knew once ya go beyond that point, you're fucked, you're gonna get strung out, you're gonna get addicted, ya know that way. (Tony, 17.4 years)

All abstainers who reported a drug history stated, in the context of the interview, that they had no future intentions regarding drug use. In fact, many former drug triers were more resolute in this regard than others who had no personal experience of drug use at the time of interview. Three of the 12 abstainers who had not tried or used illicit substances conveyed some interest in drug experimentation.

[Do you think you might ever try hash?]

Like, I'd be against it but if the opportunity came up I would probably smoke it. ... I never actually say "I'm never going to take drugs." Sometimes I think about it and then other days I say "No, it's not worth it." It depends what humor I'm in. (Jane, 15.3 years)

I probably would take a drag or something, but I wouldn't go overboard with it. You know, like just see what it's like but I wouldn't stick to it. (Helen, 16.9 years)

The majority of abstainers, however, rejected the suggestion that they might try or use a drug at any stage in the future. Fear of negative consequences was a dominant motivating factor for non-use, and the overwhelming feeling across the group was that the potentially negative consequences of drug use far outweighed any possible benefit. Although the majority expressed personal disapproval of all drug use, most did not expect others to conform with their standards and expectations. Cannabis use, in particular, was viewed with relative indifference, certainly compared with other drugs.

I'd say hash isn't as bad as some of the others because a lot of people seem to do it and they seem all right. ... Hash is the sorta drug that a lot of people would smoke but you wouldn't know it. (Jim, 18 years) 
Furthermore, most abstainers conveyed an understanding of the benefits of mood-altering substances, as indicated in their positive reports of alcoholinduced states: "It [alcohol] gives me a bit of a laugh, gives me a bit of Dutch courage. Drinking is good fun when you're out with friends. It makes the night better." The majority experienced routine exposure to drug use and expected to find themselves in situations where drugs were on offer and/or in use. Older abstainers, in particular, were well acquainted with young people who engaged in a range of drug-taking activities. It would therefore be wrong to assume that abstainers were protected from drug use by virtue of low-level exposure to the drug scene. ${ }^{9}$ However, regular drug-taking was relatively rare among abstainers' close circle of friends.

The reports of abstainers are important for a number of reasons. First, the findings suggest a pathway to becoming an abstainer: It appears that significant social and drug-related experiences precede the decision not to use drugs. In addition, a number indicated susceptibility to drug initiation. Only follow-up data can confirm or refute the possibility that some may make the transition to drugtaker at some time in the future.

Drugtakers For the majority of the study's drugtakers ( $n=21)$, daily exposure to drug use was more intense than that reported by abstainers. ${ }^{10}$ However, despite consistently high levels of drug exposure, conspicuous differences emerged in the types and levels of reported drug use. A technique known as profiling was therefore used to unravel some of the complexities of the group's drugtaking. The application of this technique led to the construction of three drug-use typologies among this relatively small sample of young drug users. ${ }^{11}$ Two discrete profiles_-"frequent" and "less frequent" drugtakerswere identified on the basis of the number of drugs tried, the quantity of drugs consumed, and the frequency of use. A third subgroup emerged from the identification of a cluster of respondents who reported significant modification of their 
drug use during their mid- to late-teenage years. Table 2 summarizes the key characteristics of "frequent" and "less frequent" drugtakers.

\section{TABLE 2 "Frequent" versus "less frequent" drugtakers}

\begin{tabular}{|c|c|}
\hline "Frequent" Drugtakers $(\mathrm{n}=11)$ & "Less Frequent Drugtakers $(\mathrm{n}=10)$ \\
\hline $\begin{array}{l}\text { Daily or near-daily cannabis } \\
\text { use. } \\
\text { Purchased a personal supply of } \\
\text { cannabis, usually by pooling } \\
\text { financial resources with friends. } \\
\text { All had experimented with and } \\
\text { regularly used a range of other } \\
\text { drugs, including Ecstasy, } \\
\text { amphetamine, LSD and } \\
\text { tranquilizers. }\end{array}$ & $\begin{array}{l}\text { Cannabis use once or twice } \\
\text { weekly. } \\
\text { Relied on friends and/or } \\
\text { situational factors for their } \\
\text { supply of cannabis. } \\
\text { Fewer had experimented with } \\
\text { or used other drugs besides } \\
\text { cannabis. Among those who } \\
\text { had, use was intermittent. }\end{array}$ \\
\hline
\end{tabular}

Descriptions offered of drug-taking events signaled considerable differences in how "frequent" and "less frequent" drug-takers used and related to drugs. For the former group, cannabis use merged, almost naturally, with routine daily events, and their accounts suggest a distinctly regular, habitual and purposeful pattern of use. In many ways cannabis use was a focal point for peer interactions and played a significant role in the group's daily activities. A strong commitment to the act of drug-taking is evident in the following account offered by one young woman:

[What lime of the day do you smoke hash?] The morning (pause). You get up and when you're walking out of your gaf [home] ya get hash handed to ya. You just go off and smoke it. ... I smoke it every day 'cos there's a few people that sells the hash at the Block and we just keep buying it. Ya see the joints just get handed to ya and ya pass them. ... I'd say I spend $£ 30$ a week on hash. (Belinda, 15 years) 
"Frequent" drugtakers ( $\mathrm{n}=11$ ) purchased a regular (usually daily) supply of cannabis - their drug of choice—by pooling financial resources with peers. Most reported a strong pattern of polydrug use as well as concurrent drug use-that is, they use two or more psychoactive substances for the attainment of heightened drug experiences. The drugs Ecstasy and amphetamine were praised for their energizing effects, and although both were strongly associated with raves and parties, several young people used these drugs in open outdoor spaces or when "buzzing around," a phrase used to denote a variable and flexible pattern of daily activities involving a high level of street-based contact with peers. In these contexts, young people were introduced to new substances and learned about their benefits, risks and effects. Friends emerged as principal advisors to young people and in many cases influenced the "move" to new drugs.

[What made you change your mind and make you feel that you'd like to try Ecstasy?] 'Cause everyone that I knew, they had been takin' E for a while, so one of them just came up to me and said "Do you want half an E?" and I was a bit hesitant at first but then I said "Go on." (Ray, 18.5 years)

Young people's accounts suggest that initiation into new drugs was frequently an informal, if not haphazard, event, one that tended not to involve advance planning. The strong influences of availability and social context were evident in most accounts, irrespective of the type of drug. Peer support and endorsement of drug-taking, coupled with the sense of security created by the presence of friends, provided ideal learning or induction settings. Collective understandings about the benefits and risks associated with drug use within a variety of settings clearly influenced drug-taking behavior. It is important to note, however, that although many informants reported drug use similar to that of their friends, there was also evidence of varied drug use among members of the same peer group. It is wrong to assume, therefore, that young people habitually complied with the drugtaking behavior of their friends. The following quotations illustrate individual 
preferences and limits irrespective of the drug choices of peers.

[What about coke?] I've never done coke ... I know people who do it and said it's a wrecker buzz. But some of me friends take coke and they need it all the time. And it's dear. I couldn't be bothered (Linda, 17.6 years)

[Do any of your friends think that drugs are dangerous?] Yeah. I get a lecture off them. . . . They always give me lectures when I'm drunk or stoned. ... I'd be sitting there wrecking their heads. [What kinds of things do they say?] "You'll have no brain cells left," they turn it into a joke and say "you're going to be sorry in the long run." (Karen, 15.8 years)

Importantly, peers also regulated the use of substances by defining the boundaries of acceptable and unacceptable drug use. It was common for respondents to say "That's not allowed" when certain drug-taking was alluded to during the course of the interview. Young people also conveyed a clear awareness of different user groups. Polydrug users who selected particular drugs for use tended to restrict or avoid contact with groups perceived to be involved in more "serious" and unacceptable drug-taking.

That's one thing that's not allowed in the door, is a junkie . . 'cos everyone is dead against it [heroin], .. . They [friends] like anything up ta E but dead against anything after that. (Sandra, 18 years)

Moreover, young people deliberately selected particular peer groups for the purpose of drug-taking. Respondents displayed an awareness of various social groupings, and several reported movement between drug-using and non-using groups of peers.

[So, you wouldn't smoke hash with those friends then?] Well, they don't mind hash like. [And what if you wanted to take E?] Like, they're not into anything like that. That's mainly why I wouldn't go out dancin' with them. I'd rather go with me other mates. (Linda, 17.6 years) 
"Less frequent" drugtakers $(\mathrm{n}=10)$ described a pattern of drug-taking that was evidently less intense. Their drug use, being incidental rather than planned, did not bear the hallmarks of deliberation. These drugtakers did not purchase a personal supply of cannabis, instead allowing situational factors to dictate use frequency. Much less time and attention was invested in drugtaking, and drug experiences were frequently reported with relative indifference. Yet these young people continued to use cannabis if and when the opportunity arose.

[How often do you smoke hash then?]

I smoke it now an' again, ya know. I wouldn't go out of me way or that now. It depends 'cos I'm not pushed. If someone has it I'll have a smoke. (Ray, 18.5 years)

Not often. I used to buy it with me cousins an' all. Now I wouldn't. If someone had it I'd say "Can I have a blow off that" ... a waste of money 'cos it does nothing for me. . . I get a better buzz off drink than off hash. (Joan, 15.9 years)

Four of the ten "less frequent" drugtakers had experimented with other drugs besides cannabis, including amphetamine, Ecstasy, and LSD. In general, however, "less frequent" drug-takers viewed other drug use besides cannabis as "too risky."

[Would you like to try E?] No. Like, that can kill ya. Ya know in the magazines, I read all about it an' all... you can die over E, ya can't over hash. (Ruth, 16 years)

Finally, the reports of a number of young people who indicated significant modification of their drug intake during the mid- to late-teenage years $(n=4)$ suggests a "maturing out" of regular drug use. All reported past daily cannabis use and had a repertoire of other drug experiences. At the time of interview, this subgroup of former "frequent" drugtakers, age 17 or over at the time of interview, had significantly reduced their cannabis intake and reported discontinued use of one or a number of substances.

Did all a that. Went through all a that. . . took E, speed, tried acid, the whole lot. Couldn't be bothered now. A waste a money and it 
wrecks ya. I just stick to me hash and a few pints now. (Ian, $J$ 7.8 years)

Across this sample of drugtakers we find diverse levels of immersion in drug use. All drugtakers, even the heaviest drug users, communicated "rules" to which they adhered. Restricted or "controlled" use of particular drugs or of a group of drugs was frequently reported. For example, many informants limited their use of Ecstasy and other "dance" drugs to parties and other social settings in an effort to minimize the perceived negative consequences of use. In other words, young people had personal limits in relation to drug use, and they reported a range of protective strategies in an effort to regulate their drug intake.

[Do you take Ecstasy every weekend?] No. No, I wouldn't, 'cos it's just ... I don't know whether you can get strung out over them or not, but I wouldn't constantly take them 'cos that'd be pushing your luck I think, anyway, pushing your luck a little bit far. (Sandra, 18 years)

The practice of selective drug avoidance was in fact widespread across the sample. For some, this involved using some drugs and rejecting others. In other instances, young people reduced their drug intake and/or restricted their use to particular settings. It is instructive to note that informal drug education-local drug "stories," peer advice, lessons from local culture and the media-informed young people's repertoire of practical knowledge about drugs and their use. For all drug-takers, cannabis use was an accepted reality or "norm." Cannabis use was not perceived to pose serious health risks and was usually equated with legal drugs, including tobacco and alcohol. On the other hand, virtually all drugtakers held extremely negative attitudes to heroin. This clear dichotomy between cannabis, on the one hand, and heroin, on the other, was a distinctive feature of their drug attitudes. Views on other drugs-Ecstasy, amphetamine, LSD, and tranquilizerswere less uniform, depending to a greater extent on the individual's range of drug experiences, with non-users more 
likely to draw attention to the immediate risk of serious injury or death. Drugtakers' accounts suggest varying levels of risk acceptability among this group of drug users who do not perceive difficulties or problems arising from their drug use.

Problem Problem drugtakers $(n=18)$ reported a range of difficulties related to their drugtakers heroin and/or other drug use. Fifteen of the 18 problem drugtakers interviewed attributed their self-reported drug problem to their heroin consumption. ${ }^{12}$ A detailed description of the group's progressive drug involvement can be found elsewhere (Mayock, 1999). This section highlights a number of the key characteristics associated with young people's "journeys" toward problem drug use.

The group described extremely early drug initiation (average age 12.4 years). Four of the 18 problem drugtakers interviewed had tried their first drug by the age of 11. Practically all left school at or before the age of 14, without any formal educational qualifications. From their early teenage years, daily life was largely unstructured, and most gradually lost contact with school-going peers and with local community-based recreational facilities. A striking feature of their reports was the rapid pace at which commitments to the drug scene developed. This integration of drug use into routine patterns of socialization and interaction coincided with a strong immersion in street culture. Drugs were easily available and provided both a legitimate and a valid response to an environment with apparently little else to offer.

We were just bored ... I'd say that had a good bit ta do with it. You're sittin' there and say "fuck sake" and then ya have a smoke and everything's new. That's the difference between being stoned and not stoned. When you're not stoned ya have nothing ta do, and when you're stoned you've lots a things ta do. (Sabrina, 18 years)

Their accounts suggest that a high level of immersion in street culture, coupled with strong incentives for use, gradually led to the acceptance of more "serious" drugs. There was 
also evidence of an attitudinal "drift" toward the endorsement of more "risky" drug trying. However, the attitudinal and behavioral transitions accompanying the "move" to heroin were not explicit or necessarily overt. On the contrary, first heroin-using events were imbued with secrecy, and virtually all respondents reported having concealed their heroin use from a number of close friends during the early stages of use.

[Was your best friend smoking heroin as well at this stage?] No. I was doing it with [pause], there was, say, me and Shane or me and Tom and me and Kevin, ya know what I mean. Like it was real "hush," like none of the rest of them knew what I was doing. (Sabrina, 18 years)

Heroin initiation typically occurred in collaboration with one or two moreexperienced users of the drug. For most, a pattern of regular heroin use developed quickly, and with this, the concealment of their heroin-related activities, particularly from friends, became difficult to sustain. Peer knowledge of the young person's heroin use emerged only gradually, and this openness facilitated the development of a regular pattern of use. This widening of the individual's social network of users provided additional access routes to heroin and other drugs, and allowed use to proceed with considerably fewer constraints than previously. Practically all of the study's heroin users reported regular use of a greater number of substances alongside their deepening involvement with opiates. Tranquilizers and street methadone, in particular, became common supplements to their heroin intake. Young people's estimations suggest that the time-lapse between first heroin use and dependence ranged from between six months and one year. In most cases the first signs of dependence came as a surprise to young people, and many attributed some of the "telling signs"-aches, pains, and fluctuating body temperature - to a "flu" or some other everyday ailment.

I didn't know I was strung out you know. I thought it was the flu or something I was coming down with. But then when I smoked the heroin, the pains would go and everything, I was back to meself. So, that's when I knew I was strung out. (Leonda, 19 years) 
At the time of interview, 12 of the 15 self-confessed opiate-dependent problem drugtakers had tried to curb their heroin intake and/or had sought treatment. ${ }^{13}$ However, an overwhelming majority related several failed attempts to "get off" heroin and/or relapse into former or more intense patterns of use. At the time of interview, all problem drugtakers reported ongoing problems related to their drug consumption. One young woman who recounted multiple unsuccessful efforts to "get off heroin described her current situation in the following terms: "There is a struggle like, every day, like not to touch it ... I haven't touched it now in a month, you just take it day by day, you know."

Drug- The combined reports of the study's abstainers, drugtakers and problem taking drugtakers illustrate the complex processes of negotiation that accompany “on the drug use and drug abstinence. Respondents' accounts-indicating move" discontinued, modified, restricted and selective use of substanceshighlight the fluidity of drug decisions. Young people were actively engaged in complex processes of assimilation and response and were aware of the range of drug options available to them. Drug decisions were subject to revision; they sometimes necessitated movement between different peer groups and invariably reflected an accumulated "wisdom" grounded in routine social experiences. A diagrammatic representation of the dominant drug-use practices to emerge from the reports of study participants is provided in Figure 1. This illustration should not be interpreted as depicting an inevitable progressive pathway from first drug use towards dependence, but as representing young people's varying styles of drug use and their transitions to elevated or decreased levels of drug involvement across time. 


\section{FIGURE 1}

Drug pathways*

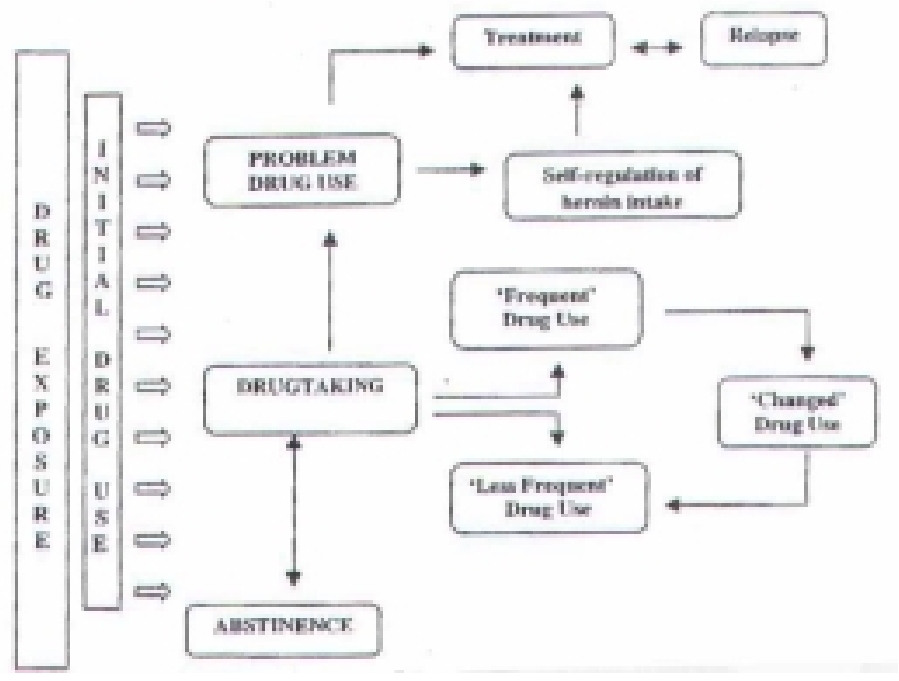

* It should be noted that this diagrammatic representation of dominant drug pathways is limited in that it is retrospective and is based on a "snapshot" in time. Follow-up data will undoubtedly contribute to the generation of more comprehensive understanding of drug transitions.

\section{Drug transitions and decisions}

Popular perceptions of drug users as passive and/or submissive have been challenged since the 1960s. Several studies (Agar, 1973; Hughes, 1961; Becker.1963; Preble \& Casey, 1969; Feldman et al., 1979) have demonstrated the purposeful role of drug use in the context of the user's lifestyle and have found drug-using behaviors to be rational when understood from the perspectives of drug users themselves. Other research has highlighted the need to view young people as motivated actors, guiding their own behavior around the use of drugs. Chein et al. (1964) reported that heroin initiates sought out established users of the drug and asked to be included in their 
drug-using activities. Shifting the focus from notions of personal predisposition to a consideration of the immediate social context of "slum life," Feldman (1968) identified strong ideological supports to becoming and remaining a heroin user and demonstrated how young people play a conscious role in their introduction into drug use. Similarly, Dembo et al. (1986), in a study of inner-city youth, found that users of first drugs selectively embedded themselves in substance-using social networks that reinforced their choice of social roles.

By focusing on processes of drug exposure, initiation and use, the findings documented in previous sections illustrate young people's active role in decision-making about the use and non-use of a range of substances. The large numbers who clearly avoid "hard" drugs (heroin and cocaine) and the numerous others who refuse all drugs despite high-level availability are in themselves indicative of decision-making in relation to drug use. Some young people avoid certain or all illicit substances, while others seek to reduce potential drug-related harm by regulating their drug intake. The collective accounts of those interviewed highlight the degree to which levels of drug involvement vary among young people who co-exist within "risky" environments. Amid the range of drug pathways identified, a number of key transitional points are particularly striking. For example, the move from non-use to use, and from cannabis to other drug use, represent two significant points of departure. The move to heroin, then, has strong symbolic significance, since the majority of young people interviewed portrayed heroin use as unacceptable. Furthermore, it appears that these critical drug transitions both relate to and communicate young people's conceptions of "soft" (cannabis), "middle" (amphetamine, Ecstasy, LSD) and "hard" (heroin, cocaine) drug use. This discussion hopes to further elucidate these distinctions, drawing attention to the situationally determined nature of risk perceptions and the ensuing effect of shifting risk boundaries on young people's drug decisions. 
Young people recognized a hierarchy of drugs and distinguished between different drugs in terms of the perceived safety versus risk of individual substances. It was common for young people to state that while they felt it was "safe" to use some drugs, others posed far more serious hazards. Across the sample, cannabis was regarded as the "safest" drug, and for the majority of study participants, cannabis use was an accepted reality or norm. However, despite abstainers' tolerance of cannabis use by friends, a large number made reference to perceived hazards, asserting that cannabis "makes you stupid," "kills brain cells," "slows you down," and "messes with your mind." Accepting that others held different views, one respondent simply stated: "They [cannabis users] say it's the lowest drug, but it's still harmful." A considerable number also believed cannabis to have addictive potential: People say ya can't get addicted to hash, but ya can. I know people who can't go a few hours without havin" a blow off a joint." The majority, however, simultaneously rejected the suggestion that cannabis use inevitably leads to other more serious drug use.

People that say that people who smoke hash move onto heroin-no. I don't think that, I think that's stupid. I know people that's smokin' hash years and years and they're not on heroin. (Vivien, 17 years)

Across the sample, the risk of "addiction" was foremost in young people's minds when assessing the potential harm associated with the use of individual drugs. However, conceptions of what constituted harmful use or dependence varied. In the following quote, for example, one of the study's drugtakers distinguished clearly between her use of cannabis and Ecstasywhich she considered to be within her control-and others' use of heroinwhich she viewed as inevitably leading to compulsive or dependent use.

I just took them [Ecstasy] for me own decision, d'ya know. I know I'd be able to stop. Like if I wanted to stop smokin' hash I could stop 'cos I tried it loads of times and I know I could stop ... 
I could stop takin' E 'cos I don't take them often. But people on heroin, they can't. (Lorraine, 16.8 years)

The notion of "control" emerged as an organizing construct, guiding how young people viewed and engaged with a range of substances. Young people distinguished between maintaining and losing control, a dichotomy closely associated with the perceived differences between needing drugs and choosing to use them. Drugtakers, for example, made frequent reference to their ability to manage and regulate their drug intake, a characteristic they did not attribute to heroin users.

I smoke hash, take E and speed because I want to, but I don't have to have it. It's not something I have ta do every day. But with gear, people on heroin, they have ta have it. (Aidan, 19.1 years)

Drugs are not important to me. I could live without them, they're not a major part of my life. The people who are on heroin, they get it just to keep them going for the day. You can probably get addicted to hash, but it's very hard. (Ray, 18.5 years)

Interestingly, problem drugtakers made similar distinctions, and several referred to a previous belief in their ability to retain control as factor influencing the "move" to heroin.

Everyone says "I won't get strung out, I know when to stop," everyone says that. Fuckin' hell! "Ah, I won't get strung out 'cos I'm not like that" . . . When I started smokin' [heroin] like I was sayin' "I can control this," but you can in your bollocks. (Sabrina, 18 years)

Yeah, it [heroin] kept me occupied until I got the bang like, that I needed it. I was takin' it out of choice at the time and then it became a need. (Alan, 19.5 years)

Drug decisions hinged largely on the perceived boundaries of "acceptable risks." Beliefs about what constituted "safe" versus "risky" behavior varied across the sample, and some young people were clearly prepared to take greater risks than others. 
[When you took E the first time, did you know much about it?]

Yeah, I did, and 1 knew the risks of it and I just wanted to try it. (Sandra, 18 years)

I don't think there's anything dangerous about hash ... I could get speed easily now if I wanted to, but I wouldn't try it. And E, that's one thing I would never try ... too many people die from that. It just goes straight to their heads and they die. (Crystal, 15.5 years)

Young people invariably described their views on individual substances with reference to other drugs. Dominant drug attitudes, and beliefs about the use of a range of drugs, strongly suggest that risks were considered and calculated in relative terms. Young people's assessments, being conditional rather than unconditional, were contingent on a range of considerations, with the circumstances or "setting" of use featuring strongly in appraisals of danger and risk.

It's [cannabis] harm but it's no harm like. It's harm in a way 'cos it's a drug. It's no harm 'cos it's not as bad as the rest a them like. (Mark, 17.4 years)

I know all drugs are dangerous except hash. Hash is nothing. That should be legalized. I know E is dangerous .... it's not even the E, it's the company you're with. I really do think that 'cos I was at a few parties, now that was great and nothing bad ever happened. And I was at another party one night and it was bollocks, the company was crap. (Lorraine, 16.8 years)

At the time of trying a new drug - be it cannabis. Ecstasy, amphetamine or heroin - the boundaries of acceptable drug use shifted to accommodate newly defined benefits and risks. ${ }^{14}$ In the case of Ecstasy, it was noticeable, for example, that previous fears were dismissed or forgotten as familiarity with the drug increased. This kind of learning was a critical factor, in many cases, in the transition from cannabis to other drug use.

[Did you want to try Ecstasy straight away then?] No, 'cause I didn't know what they were or what to expect from them. And I was afraid as well. But when people became familiar with them I tried it just to see what it was like. (Janice, 18 years) 
Social processes related to peer-group interaction appear to have a strong role in the transition to new drugs. This is clear from the numerous descriptions offered of drug-use scenarios involving the presence of friends. Importantly, however, young people did not interpret the role of peers as "pressure" to engage in drug use. This distinction between perceived "pressure" and "preference," within particular settings and contexts, is important, indicative of the need to consider social processes other than peer pressure in the development and maintenance of drug "careers."

I only do it [take drugs] if I want to do it. Like, nobody ever forced me to do it I only do it if I feel like it, if I want to do it ... it depends on the humor I' $m$ in like. (Mark, 17.2 years)

I wanted to do it [smoke heroin]. They [friends] didn't want to give it to me, but I'd have got it somewhere else otherwise. I'd have got it off someone. (Sylvia, 18.6 years)

For the majority of problem drugtakers, the acceptance of more precarious drug-taking extended, albeit gradually, to heroin. Most stated that they previously held negative attitudes to heroin and were aware of at least some of the potential risks at the time of initiation. Most had also received warnings from both parents and like-aged peers. The accounts of several suggest, however, that the warnings they received (emphasizing, in most cases, a downward spiral towards dependence) did not match their personal observations and experiences. It should be emphasized that most were strongly immersed in the local drug scene at this juncture and had a lengthy repertoire of drug experiences.

Where we hung around there was a couple that were on heroin, but I (pause) ... they never seemed to have problems with it, you know. They were a year or two older than us and they never seemed to have problems or to be strung out, so .... (Gerald, 19 years)

The distinction between smoking and injecting heroin and the perception that inhalation was a relatively "safe" means of administration, certainly compared with intravenous use, provided further impetus and justification for use. 
Like we thought that smoking it wasn't dangerous, that needles was . . I'm only smoking it. I was thinking like, it's not really that bad so long as you don't go near the needle. (Connie, 17.4 years)

The processes surrounding young people's acceptance of more risky drugtrying are complex and cannot be attributed solely to stable or enduring cost-benefit analyses on the part of individuals. This is clear from the numerous accounts suggesting social and contextual influences on drugtaking. Moreover, for use to proceed beyond experimentation, drug-taking had to be perceived as rewarding. It is interesting to note, in this context, that many former drug triers categorized as abstainers did not perceive drug use to be personally fulfilling; "I didn't like it [cannabis]. It was a real dopey buzz, ya know." Others, who modified their drug use as time progressed, appeared to have redefined the benefits of particular drugs.

I got a big mad turn off it [cannabis] and I just don't smoke it much anymore ... Whereas before I was smokin' it all day and all night. You know, when you're smokin' it a lot you just get sick of it ... [So, you don't smoke it as much now?] No, not really at all. If I was having a few drinks and someone handed me a joint, then I'd probably take a few blows off it, that's all. (Janice, 18 years)

The complex social and interpersonal dynamics surrounding drug use involve negotiation and renegotiation across time. Drug use, like many behaviors, changes as young people progress through the teenage years. Young people in the current study conveyed a range of practical knowledge about drugs, knowledge acquired largely through personal experience and routine social interaction. This socially distributed information played an influential role in their drug decisions, as did the perceived risks versus rewards associated with drug-taking. Perceptions of risk susceptibility and acceptability emerged as important components of a complex array of factors, operating at both individual and group levels, to produce varied responses to a social milieu characterized by high availability of drugs and exposure to them. 


\section{Discussion and conclusion}

This paper has attempted to extend our social understanding of drug use within "high risk" localities by drawing attention to the range of drug options available to young people who experience high exposure to drugs and the drug scene. The findings illustrate a diverse range of drug-taking behaviors and suggest that a large number of young people growing up in areas where drug use is concentrated will experiment with and use drugs at some level. Informants' experience and use of illegal drugs was well in excess of national norms for this age group. Across the sample, drug use ranged from occasional or moderate use through to regular, heavy and problematic levels of drug involvement. This finding in itself is indicative of the range of drug choices available to young people. The large number reporting intensive mixed polydrug use is of particular concern. However, it is a documented experience - and clearly the case in the current study - that many young drug users are unlikely to perceive their drug use as problematic (Lloyd, 1998; Parker et al., 1998; Verees, 2000). While all of the study's informants associated heroin consumption with a range of problems, other drug use - in particular, cannabis use-was not perceived to have serious negative health consequences. For the majority, being "on drugs" was an expression attributed only to heroin users.

The young people interviewed live amid a thriving drugs culture and indicated varying degrees of association and involvement with the local drug scene. The negotiation of this eminently "risky" environment was influenced by a complex interplay of social/contextual factors and was strongly mediated by young people's experience of, and interaction with, the social environment (Mayock, 2000a). A range of socially transmitted rules and expectations concerning the use and non-use of substances influenced individual drug-taking practices, as did their perceptions of risk versus reward. Young people's perspectives on a range of drugs were grounded in 
the cultural "lessons" transmitted through routine social interaction. These lessons influenced the customs and "styles" of use as well as the benefits ascribed to drug use. A wide range of situational and social factors influence perceptions of and responses toward risk (McKeganey \& Barnard, 1992; Rhodes \& Quirk, 1995, 1996). It follows that individual responses to risk cannot be divorced from lifestyle characteristics, peer groups, and social and community norms and expectations.

Previous research on drug-related decision-making has drawn attention to the importance of risk boundaries and has found that young people with similar drug-related experiences appear to share the same basic "rules" (Boys et al., 1999; Glassner \& Loughlin, 1987). The current study's emphasis on how risk is lived through interaction and experience helps to further our understanding of drug-related decision-making by drawing attention to the link between individual risk perceptions, drug-use contexts, and the negotiations that characterize interaction within these contexts. Perceptions of risk and beliefs about risk susceptibility (and invulnerability) varied, both between and within the three categories of research participants, and the boundaries and limits governing drug use at various junctures differed for different young people. As Rhodes (1995: 128) points out:

What is perceived to be a cost, a benefit, or a risk is not static or necessarily shared among all individuals alike - be they drug users or epidemiologistsbut is situated within different social contexts of beliefs and behavior.

For some young people in the study, the notion of all drug use acted as a risk boundary. For others, some drugs were viewed as acceptable and relatively safe, while others were considered to pose too high a risk. Yet others, who extended their drug repertoires to include opiates, redefined previous boundaries so that behaviors once perceived to be too risky gradually became normalized and acceptable. The negotiation and renegotiation of risk boundaries was highly situational, inextricably bounded by context and influenced by processes 
of social interaction, communication, and routine experiences. The context of use clearly played an important role in drug decisions, illustrating how "choice" is influenced, shaped, and constrained by the everyday realities and experiences of social life.

In common with the findings of other research on drug use by young people-in particular, those arising from the use or the integration of qualitative methodologies (Glassner \& Loughlin, 1987; Coffield \& Gofton, 1994; Measham et al., 1998) — the current study highlights the critical capacities of young people and the role of individual assessments of risk in the decision to use drugs. Moreover, it would appear that within "high risk" environments drug decisions are often not fundamentally about whether or not to take drugs, but focus instead on acceptable versus unacceptable drugs and on perceived appropriate versus inappropriate styles of use. Practically all respondents distinguished between different levels of drug involvement, and they did not consider all drug use per se to be inherently damaging or problematic. Young people's drug attitudes, behaviors, risk perceptions, and motives for using or for not using individual substances provide important insights into the lived experience of "risky" environments.

It would appear that those who aim to intervene in the lives of young people to prevent or delay drug initiation and/or movement to new drugs seek to engage a "moving target." Drug transitions can be rapid and changeable and are clearly difficult to predict. The importance of social settings and contexts, and the influential role of situational factors in movements "into" and "out of new drugs and "away from" former patterns of use have been highlighted. The young people interviewed conveyed a sophisticated, socially generated awareness of drug risk. Furthermore, they themselves implemented a range of strategies in an effort to reduce the harmful consequences of drug use. This finding highlights the strengths that can be drawn upon in efforts to reduce the damaging effects of drug consumption. It is interesting to note, in this 
context, that there is no firm commitment to anything more than "consideration" of harm-reduction strategies in the Irish government's Second Report of the Ministerial Task Force on Measures to Reduce the Demand for Drugs (1997). The recently launched National Drugs Strategy (2001) maintains a strong emphasis on primary prevention. While acknowledging the limited efficacy of "just say no" messages and recommending an approach to prevention that seeks "to equip young people and other vulnerable groups with the skills to make informed choices about their health, personal lives and social development" (p. 5), no commitment is made to the development or implementation of strategies aimed at encouraging risk-reduction among young people. ${ }^{15}$ This is despite clear evidence of an expansion and diversification of drug-use practices among the young. While Irish drugs policy has gradually moved toward a broader and more differentiated view of drugs and their use, it is in danger of being left behind by young people's increasingly diverse and sophisticated understandings of drug-related risk. It would appeal-that young people's views on "risk" are not grounded in the lessons learned from ominous warnings, but in the messages transmitted during the course of routine social encounters and negotiations within a range of everyday settings. The social and cultural contexts of young people's lives clearly need to be acknowledged within a range of strategies aimed at reducing the likelihood of serious and damaging forms of drug use. Furthermore, formal acknowledgment and fostering of the critical capacities of young people is likely to have more to offer future preventive strategies, than approaches that assume uncritical and indiscriminate behavior around drugs on the part of young people.

Notes

1. The 1990s marked a clear departure from previous decades, during which adolescent drug use was a minority activity (Nevin et al., 1971; Shelley et al., 1982), and signaled increased contact with and use of illicit substances by young people. 
2. Compared with cannabis use in other participating European countries (30 in total), the use of cannabis in Ireland was twice as common as the average for all other countries (32\% vs. $16 \%$ ), and the use of illicit drugs other than cannabis was slightly above average (9\% vs. 6\%) (Hibell et al., 2000).

3. This concentration of attention on "high risk" drug-use categories, most notably heroin and other intravenous drug use, can be traced to the opiate epidemic of the 1980s and the public health crisis of HIV transmission (Butler, 1991).

4. During the second phase of fieldwork, 42 of the study's original 57 research participants were contacted and re-interviewed.

5. Due to limitations of space, this paper does not report on data from focus-group discussions.

6. It is important to note that in the study the term drug is used to refer to solvents, inhalants, cannabis, amphetamines. Ecstasy, hallucinogens, tranquilizers, cocaine and opiates, most of which are regarded as illicit drugs and/or are procured by illicit means. Tobacco and alcohol use are referred to independently throughout the research.

7. The majority of young people were anxious to conceal their drug use from adults. However, there were many instances when young people smoked cannabis unreservedly in my presence. Other young people (usually over the age of 17 years) reported that their parents and/or siblings were aware of their cannabis use.

8. Lifetime drug use refers to the drugs ever tried or used by study participants and is not indicative of the frequency or regularity of use.

9. In addition to casual drug encounters, a large number of abstainers had first-hand experience of serious drug use, in some cases within their immediate families. Five reported that an immediate family member (either a parent or a sibling) experienced drug-related problems, and six others stated that a more distant family member (aunt, uncle, cousin) had a "heroin problem." Hence, in excess of $60 \%$ of the total sample of abstainers had direct contact with individuals who experienced drug-related difficulties.

10. Drugtakers reported daily or near-daily exposure to drugs and drug users in the context of their largely street-based leisure activities. Drug consumption had all the appearances of being an integral part of street culture.

11. Typologies are a useful device in the organization of qualitative data. They are a means of categorizing events or people without necessarily involving a sense of progression from one event to another (Scale \& Kelly, 1998). 
12. The three remaining self-declared problem drugtakers reported problems associated with other drugs: one young man stated that he was "addicted" to inhalants; one young woman described problems related to her amphetamine and other stimulants use; one respondent stated that she experienced problems related to her intake of cannabis and tranquilizers. Since opiates were the most commonly stated drug of "misuse" across the study's sample of problem drugtakers, this section focuses primarily on the "move" to heroin.

13. It was common for young people to purchase street methadone in an effort to selfregulate their heroin intake.

14. The degree to which cannabis use was "normalized," even among non-users of the drug. suggests that this particular drug transitions did not carry the symbolic import of other drug transitions.

15. This reluctance by policy makers to explicitly endorse harm-reduction strategies has similarly been noted in the British context (Wibberley \& Price, 2000).

References Advisory Council on the Misuse of Drugs (ACMD) (1998). Drug Misuse and the Environment. London: HMSO.

Agar, M. (1973). Ripping and Running: A Formal Ethnography of Urban Heroin Users. New York: Academy Press.

Becker, H.S. (1963). Outsiders: Studies in the Sociology of Deviance. London: Free Press.

Bell, R., Pavis, S., Cunningham-Burley, S. \& Amos, A. (1998). Young men's use of cannabis: Exploring changes in meaning and context over time. Drugs: Education, Prevention and Policy, 5, 2. 141-155.

Boys, A., Marsden, J., Fountain. J., Griffiths, P., Stillwell, G. \& Strang, J. (1999). What influences young people's use of drugs? A qualitative study of decision-making. Drugs: Education, Prevention and Policy, 6, 3, 373-387.

Boys, A., Marsden, J. \& Strong, J. (2001). Understanding reasons for drug use amongst young people: A functional perspective. Health Education Research, 16, 4, 457-469.

Butler, S. (1991). Drug problems and drug policies in Ireland: A quarter of a century reviewed. Administration, 39, 3, 210-233.

Chein, I., Gerard, D., Lee, R.S. \& Rosenfeld, E. (1964). The Road to H. New York: Basic Books. 
Coffield, P. \& Gofton, L. (1994). Drugs and Young People. London: Institute for Policy Research.

Comiskey, C. (1998). Estimating the Prevalence of Opiate Use in Dublin, Ireland, during 1996. Dublin: Department of Health and Children.

Crum, R.M., Lillie-Blanton, M. \& Anthony, J.C. (1996). Neighborhood environment and opportunity to use cocaine and other drugs in late childhood and adolescence. Drug and Alcohol Dependence, 43, 155-161.

Cullen, B. (1991). Community and Drugs: A Case Study in Community Conflict in the InnerCity of Dublin. Unpublished M.Litt. thesis, Department of Social Studies, Trinity College, Dublin.

Dean, G., Bradshaw, J. \& Lavelle, P. (1983). Drug Misuse in Ireland, 19S2-19S3. Investigation in a North Central Dublin Area and in Galway, Sligo and Cork. The Medico Social Research Board.

Dembo, R., Blount, W.R., Schmeidler, J. \& Burgos, W. (1986). Perceived environmental drug use risk and correlates of early drug use or nonuse among inner-city youths: The motivated actor. International Journal of the Addictions, 21, 977-1000.

Feldman, H.W. (1968). Ideological supports to becoming and remaining a heroin addict. Journal of Health and Social Behaviour, 9, 131-139.

Feldman, H.W., Agar, M. \& Breschner, G.M. (eds.) (1979). Angel Dust: An Ethnographic Study of PCP Users. Lexington, MA: Health/Lexington Books.

First Report of the Ministerial Task Force on Measures to Reduce the Demand for Drugs, 1996. Dublin: Department of the Taoiseach.

Fountain. J., Bartlett, H., Griffiths, P., Gossop, M., Boys, A. \& Strang, J. (1999). Why say no? Reasons given by young people for not using drugs. Addiction Research, 1,4, 339-353.

Glassner, B.G, \& Loughlin, J. (1987). Drugs in Adolescent Worlds: Burnouts to Straights. London: Macmillan.

Hawkins, D.J., Catalano, F. \& Miller, Y. (1992). Risk and protective factors for alcohol and other drug problems in adolescent and early childhood: Implications for substance abuse prevention. Psychological Bulletin, 112, 1, 64-105.

Hibell, B., Andersson, B., Ahlstrom, S., Balakireva, O., Bjarnasson, T., Kokkevi, A. \& Morgan, M. (2000). The 1999 ESPAD Report: Alcohol and Other Drug Use Among Students in 30 European Countries. Stockholm: The Swedish Council for Information on Alcohol and Other Drugs (CAN), The Pompidou Group at the Council of Europe. 
Hughes, P. (1961). The Fantastic Lodge: The Autobiography of a Drug Addict. New York: Fawcett World Library.

Kandel, D.B. \& Logan, J.A. (1984). Patterns of drug use from adolescence to young adulthood: 1. Periods of risk for initiation, continued use, and discontinuation. American Journal of Public Health, 74. 7. 13-40.

Lloyd, C. (1998). Risk factors for problem drug use: Identifying vulnerable groups. Drugs: Education, Prevention and Policy, 5, 3, 217-232.

McKeganey, N. \& Barnard, M. (1992). AIDS. Drugs and Sexual Risk. Buckingham: Open University Press.

McKeown, K.. Fitzgerald, G. \& Deegan, A. (1993). The Merchant's Quay Project: A Drugs/HIV Service in the Inner City of Dublin, 1989-1992. Dublin: Kieran McKeown Limited, Social and Economic Research Consultants.

Mayock, P. (1999). Young People and Drugs: The Social Experiences of Young People in an Inner-City Dublin Community. Unpublished report. Addiction Research Centre. Trinity College, Dublin.

Mayock, P. (2000a). Choosers or Losers? Influences on Young People's Decisions About Drugs in Inner-City Dublin. The Children's Research Centre, Trinity College, Dublin.

Mayock, P. (2000b). Engaging "difficult-to-reach" young people in a study of inner-city drug use. In J. Fountain (ed.). Understanding and Responding to Drug Use: The Role of Qualitative Research. Lisbon: EMCDDA Monograph.

Measham, F., Newcombe, R. \& Parker, H. (1994). The normalization of recreational drug use amongst young people in North-West England. British Journal of Sociology, 45, 2, 287-312.

Measham, F., Parker, H. \& Aldridge, J. (1998). Starling. Switching, Slowing and Slopping. SPARC: Department of Social Policy and Social Work, University of Manchester.

National Drugs Strategy (2001). Ireland's National Drugs Strategy. 2001-2008. Dublin: The Stationery Office.

Nevin, M., O'Rourke. A., Wilson-Davis, K. \& Dean, G. (1971). Drugs - a report on a study of Dublin post-primary schoolchildren. 1970. Journal of the Irish Medical Association, 64, 91-100.

O’Brien, M., Moran, R., Kelleher, T. \& Cahill, P. (2000). Statistical Bulletin, 1997 and 1998. Dublin: The Health Research Board. 
O'Higgins, K. (1996). Treated Drug Misuse in the Greater Dublin Area: A Review of Five Years, 1990-1994. Dublin: The Health Research Board.

O’Higgins, K. \& Duff, P. (1997). Treated Drug Misuse in Ireland: First National Report. Dublin: The Health Research Board.

O'Kelly, R., Bury, G., Cullen, B. \& Dean, G. (1988). The rise and fall of heroin use in an inner-city area of Dublin. Irish Medical Journal, 157, 2, 35-38.

Parker, H., Aldridge, J. \& Measham, P. (.1998). Illegal Leisure: The Normalization of Adolescent Recreational Drug Use. London: Rout-ledge.

Parker, H., Bakx. K. \& Newcombe, R. (1988). Living with Heroin: The Impact of a Drugs "Epidemic" on an English Community. Manchester: Open University Press.

Pearson, G., Gilman, M. \& McIver, S. (1985). Young People and Heroin: An Examination of Heroin Use in the North of England. London: Health Education Council. Research Report No. 8.

Preble, E. \& Casey, J. (1969). Taking care of business: The heroin user's life on the street. International Journal of the Addictions, 4, 1 -24.

Rhodes, T. (1995). Theorising and researching "risk": Notes on the social relations of risk in heroin users' lifestyles. In P. Aggleton, P. Davies \& G. Hart (eds.), AIDS: Safety, Sexuality and Risk. London: Taylor \& Francis.

Rhodes, T. (2000). The multiple roles of qualitative research in understanding and responding to drug use. In J. Fountain (ed.). Understanding and Responding to Drug Use: The Role of Qualitative Research. Lisbon: EMCDDA Monograph.

Rhodes, T. \& Quirk, A. (1995). Theorising and researching risk: Notes on the social relations of risk in heroin users' lifestyles. In P. Aggleton, G. Hart \& P. Davies (eds.), AIDS, Safety. Sexuality and Risk. London: Taylor \& Francis.

Rhodes, T. \& Quirk, A. (1996). Heroin, risk and sexual safety: Some problems for interventions encouraging community change. In T. Rhodes \& R. Hartnoll (eds.), AIDS, Drugs and. Prevention: Perspectives on Individual and Community Action. London: Routledge.

Seale, C. \& Kelly, M. (1998). Coding and analysing data. In C. Seale (ed.), Researching Society and Culture. London: Sage Publications.

Second Report of the Ministerial Task Force on Measures to Reduce the Demand for Drugs, 1997. Dublin: Department of the Taoiseach. 
Shelley, E.B., Wilson-Davis, K. \& O'Rourke, F. (1982). Drugs-a study of Dublin postprimary schools. Irish Medical Journal, 75, 7, 254-259.

van Meter, K.M. (1990). Methodological and design issues: Techniques for assessing the representatives of snowball samples. In E.Y. Lambert (ed.), The Collection and Interpretation of Data from Hidden Populations. NIDA Research Monograph 98.

Verees, K. (2000). Drug use among young detainees in Hungary. In A. Springer \& A. Uhl (eds.). Illicit Drugs: Patterns of Use-Patterns of Response. Proceedings of the 10th annual ESSD Conference on Drug Use and Drug Policy in Europe. Innsbruck: Studien Verlag.

Watters, J.K. \& Biernacki, P. (1989). Targeted sampling: Options for the study of hidden populations. Social Problems, 36, 4, 416-430.

Wibberley, C. \& Price, J. (2000). Patterns of psycho-stimulant drug use amongst "social/operational users": Implications for services. Addiction Research, 8, 95-111. 